Service social

\title{
Problème social : le concept et les principales écoles théoriques
}

\section{Robert Mayer et Marcelle Laforest}

Volume 39, numéro 2, 1990

Les problèmes sociaux

URI : https://id.erudit.org/iderudit/706475ar

DOI : https://doi.org/10.7202/706475ar

Aller au sommaire du numéro

Éditeur(s)

École de service social de l'Université Laval

ISSN

1708-1734 (numérique)

Découvrir la revue

Citer cet article

Mayer, R. \& Laforest, M. (1990). Problème social : le concept et les principales écoles théoriques. Service social, 39(2), 13-43. https://doi.org/10.7202/706475ar

\section{Résumé de l'article}

Ce texte, de nature théorique, aborde la définition du concept de problème social et fait une présentation des principaux courants analytiques ou courants de pensée qui prévalent actuellement dans l'analyse sociologique des problèmes sociaux. Les auteurs y présentent tour à tour : l'École de Chicago, le modèle culturaliste, l'approche fonctionnaliste, l'approche critique du conflit social, l'interactionnisme symbolique et les théories de la réaction sociale et de l'étiquetage et, finalement, le constructivisme. 


\title{
ARTICLES
}

Robert Mayer, professeur à I'École de service social, Université de Montréal.

Marcelle Laforest, professeure à

l'École de service social, Université Laval.

\section{Problème social : le concept et les principales écoles théoriques}

\author{
Robert Mayer \\ Marcelle Laforest
}

D'usage courant dans la vie quotidienne, la notion de " problème social " demeure encore imprécise dans les diverses disciplines des sciences humaines. Et ce, malgré un nombre considérable de publications sur les problèmes sociaux (articles, livres et surtout recueils de textes ou " readers »); malgré aussi l'existence, aux ÉtatsUnis, d'une société pour l'étude des problèmes sociaux, l'American Association for the Study of Social Problems, et sa revue spécialisée, Social Problems. De fait, l'enseignement, la recherche et les publications sur les multiples problèmes sociaux qui confrontent les sociétés actuelles relèvent $d$ 'une longue tradition surtout américaine. En dépit de cet effort, l'imprécision demeure, notamment parce que la notion de problème social a toujours été fort discutée mais les définitions ont varié dans le temps. II faut donc d'emblée admettre que l'analyse des problèmes sociaux n'est pas, elle-même, sans problème ! En effet, parler de problème social, c'est déjà, en soi, comme l'ont noté plusieurs auteurs, poser un problème sociologique (Laskin, 1965).

Toute société, nous dit R. Rezsohazy, fait face à des problèmes sociaux; s'ils sont reconnus, "ils deviennent des enjeux et alors des 
acteurs et des groupes sociaux se mobilisent pour participer à ces enjeux " (1980:81). C'est dans la logique de cette dynamique que les problèmes sociaux ont, dès ses origines, constitué la principale cible d'intervention du service social : "Depuis leurs tout débuts vers la fin du $19^{e}$ siècle, les services sociaux se sont surtout attaqués aux problèmes les plus flagrants des individus et des familles: la pauvreté, la misère sous toutes ses formes, les handicaps physiques et mentaux sont devenus la cible principale de leurs interventions " (Perron, 1986 : 34). Mais, s'est demandé R. Barbier (1978), I'enseignement des sciences humaines permet-il à l'intervenant social de comprendre quelque chose à la complexité des problèmes sociaux ?

Non, répond-il, car les sciences humaines cherchent moins à expliquer la réalité sociale qu'à " circonscrire, marquer et finalement contrôler la clientèle cible des travailleurs sociaux : la classe ouvrière urbaine dans ses composantes économiques, socio-affectives et socio-culturelles" (1978: 34). D'ailleurs, affirme-t-il, " on cherchera en vain un seul ouvrage didactique en sciences humaines à destination du travail social qui apporterait une lumière plus appropriée à l'explication scientifique des faits quotidiens brassés par les praticiens du travail social » (1978: 34$)$. Plus récemment, Martin et Chopart (1988: 86) se sont plaints d'un manque de réflexion approfondie en travail social sur le concept de problème social. M. Tachon (1985 : 181) estime de son côté que le travail social tente de s'imposer de plus en plus comme secteur spécialisé dans la gestion des problèmes sociaux, mais qu'il s'agit d'un secteur en crise. C'est donc dire que le défi est de taille lorsqu'il s'agit de préciser le concept de problème social, même s'il est relativement facile de le circonscrire à travers des situations concrètes telles que la pauvreté, le chômage, etc. Toutefois, dans l'Angleterre du $17^{\mathrm{e}}$ siècle, les taudis, la pauvreté, la délinquance et la maladie mentale n'étaient pas considérés par l'ensemble de la population comme des problèmes sociaux (Merton et Nisbet, 1961 : 5). Ceci doit donc nous amener à nous interroger plus sérieusement sur la définition du problème social, en nous intéressant moins à l'objet réel et concret constitué par un phénomène social particulier (délinquance, maladie mentale ou autre) qu'au concept lui-même et à l'évaluation des perspectives théoriques d'analyse. Le présent article a donc pour objet, dans un premier temps, d'apporter des précisions sur la notion de problème social, suite à quoi nous ferons une brève revue des principaux courants d'analyse sociologique des problèmes sociaux depuis le début du vingtième siècle. 


\section{La notion de problème social}

Selon R. Lenoir, la notion de problème social renvoie historiquement à deux conceptions principales : "La première recouvre assez bien le champ dont traitent "l'aide sociale " (les pauvres, les cas "sociaux", les marginaux, etc.), la " sécurité sociale»(la vie hors travail, notamment la vie de famille et celle des personnes âgées, etc.), bref, des problèmes auxquels sont confrontés professionnellement les travailleurs "sociaux" (assistantes "sociales", éducateurs spécialisés, etc.) et que visent à résoudre les politiques et les lois "sociales". La seconde acception provient d'un autre sens que ce terme avait déjà au $19^{\mathrm{e}}$ siècle : proche de celui de "socialisme ", de «question sociale » ou « d'enquête sociale» (1989: 57). Cet article se réfère surtout à la première conception du terme.

Nous ne procéderons pas à un relevé exhaustif des différentes définitions de la notion de problème social; cela a été fait plusieurs fois avant nous (voir références) et la plupart des définitions se ressemblent. Nous en examinerons plutôt quelques-unes parmi les plus usuelles ou les plus "classiques", afin d'en retirer les éléments principaux. Pour ce faire, nous puiserons à même la documentation américaine, européenne et québécoise en suivant une logique historique d'exposition.

Pour la période précédant les années 1960, nous utiliserons l'analyse de C.W. Mills (1963) portant sur le contenu de plusieurs dizaines de livres constitués de morceaux choisis ("textbooks") publiés entre les années 1920 et 1960 et traitant des problèmes sociaux aux États-Unis. Mills souligne que l'approche traditionnelle vis-à-vis les problèmes sociaux est essentiellement a-théorique et a-historique par la préférence accordée à la perspective descriptive, empiriciste et a-politique par son incapacité à dépasser le niveau individuel des problèmes (cf. le psychologisme) pour aborder celui des structures sociales. Dans son très beau livre intitulé L'imagination sociologique (1968 : 13), il avança donc quelques propositions pour renouveler I'analyse des problèmes sociaux. On lui doit cette distinction des plus fructueuses entre les «épreuves personnelles » qui surgissent au sein du caractère de l'individu et affectent ses rapports immédiats avec autrui, et les "enjeux collectifs" qui soulèvent des questions transcendant le voisinage de l'individu et le champ de sa vie intérieure. Pour C.W. Mills, les analyses concernant ces deux niveaux doivent se compléter et non s'opposer.

Dans la même décennie, Merton et Nisbet (1961: 700) avançaient qu'un problème social consiste en un écart entre ce qui est et ce que les gens pensent devoir être (ou encore, entre les conditions actuelles et les valeurs et normes sociales), écart considéré corrigible. Fait 
intéressant qui mérite d'être souligné dès à présent, les administrateurs sociaux et les tenants de l'épidémiologie des années 1980, tels Pineault et Daveluy (1986) ou De Robertis et Pascal (1987), abordent la notion de problème social dans le même sens. Pour les premiers, par exemple, la notion de problème exprime "l'écart ou la différence entre un état optimal, défini de façon normative, et l'état actuel ou réel » (Pineault et Daveluy, 1986 : 76). La même perspective s'affirme chez N. Cohen et al. (1964), à qui les problèmes sociaux apparaissent comme des problèmes de valeurs pouvant être cernés à la lumière de ce qu'une société juge être bien ou mal. Selon eux, un problème social doit être analysé en relation avec les systèmes de valeurs auxquels adhèrent les individus et les groupes sociaux. Et si nous acceptons la relativité des valeurs, on se trouve donc en présence de plusieurs définitions d'un problème social selon les individus et les groupes impliqués (voir plus loin).

Ces définitions vont rapidement trouver écho au Québec. Ainsi par exemple, P. Durocher précise que «les problèmes sociaux doivent être distingués des autres problèmes par l'étroite relation que ces problèmes entretiennent avec les contextes institutionnels et normatifs d'une société; un fait social est dit problème en ce sens qu'il représente une interruption, une brisure dans l'ordre des choses conçu comme normal et valable par une société » (1965: 160). Même analyse chez A. Normandeau qui ajoute : ces problèmes "sont sociaux en ce sens qu'ils appartiennent au domaine des relations humaines [...]. Ils sont des problèmes en ce sens qu'ils représentent des coupures au sein du «train-train quotidien » accepté ou désiré; violations du "bon " ou du " correct", telles que la société définit ces qualités; dislocations au sein des modèles et des relations sociales que la société préfère » (1966: 19). On retrouvera ces mêmes notions de dislocation et de dysfonction du système social quelques années plus tard chez Kallen et Miller (1968), Lenoir (1989) et Perron (1986); pour ce dernier, la dysfonction est plus spécifiquement l'écart entre les normes d'une société et le comportement d'un nombre plus ou moins grand d'individus (1986:65).

Un certain nombre d'auteurs tels Horton et Leslie (1971), Manis (1974a), Laskin (1965), Lalonde (1976:43) et Beaudoin (1987: 16) précisent quelques dimensions servant de base à l'identification d'un problème social : il y a problème lorsqu'un grand nombre de personnes sont affectées par une situation donnée, que cette situation est jugée intolérable, et que les gens sont conscients de la nécessité d'une action collective. En ce sens, " aucune situation, peu importe combien dramatique elle puisse être, combien choquante pour autrui, n'est un problème social à moins que les valeurs d'un nombre considérable de gens ne la définissent comme un problème " 
(Horton et Leslie, $1971: 5$ ). Les problèmes sociaux sont donc, affirme Laskin, des problèmes de valeurs (1965:7).

R. Blum adopte également cette perspective en soulignant que les problèmes sociaux présentent les caractères d'une crise sociale, " en ce sens que leurs conséquences atteignent l'ensemble du groupe ou de la société et doivent être traitées par des mesures de caractère collectif » $(1970: 40)$. De plus, les problèmes sociaux ne sont pas des phénomènes "anormaux", bien au contraire, ils apparaissent « comme les manifestations logiques, compréhensibles et inévitables des structures sociales, des valeurs et des comportements en usage » $(1970: 42)$. Conséquemment, ajoute-t-il, il n'est pas fondé de soutenir « que la responsabilité des problèmes sociaux incombe à des individus moralement inférieurs » (Idem).

Après avoir montré, avec plusieurs exemples à l'appui, la "versatilité " de la conscience collective à propos de la perception des problèmes sociaux, M. Pelletier (1978) insiste de son côté pour dire que ces derniers naissent des " contradictions internes" (1978: 189) et que conséquemment, les débats autour des problèmes sociaux sont le résultat d'un jeu complexe de rapports de force au sein de la société. La reconnaissance sociale du problème, indispensable à son accession au statut de problème social, renvoie directement à la question du pouvoir et des rapports de force entre "ceux qui subissent les problèmes de bien-être et ceux qui détiennent le pouvoir de mettre en place des politiques sociales c'est-à-dire ceux qui contrôlent l'appareil étatique " (1978: 166). Bref, tout problème social est aussi un problème éminemment politique. De plus, les problèmes sociaux comportent également une dimension économique, reliée plus ou moins directement à l'insuffisance du revenu. Les problèmes sociaux ne sont donc pas de nature exclusivement humanitaire mais comportent des dimensions politiques et économiques qui sont le plus souvent masquées (1978: 183).

Pour R. Rezsohazy, les problèmes sociaux sont presque toujours associés au changement social : " Le problème social pointe ou surgit au cours du changement; trois notions permettent de le comprendre : la rupture, le défi, l'inadéquation [...] Au départ, il y a toujours une rupture entre un état qui s'est formé et une attente. Cette situation lance un défi. Celui-ci apparait comme une menace ou un danger pour l'altérité ou la sécurité ou le bien-être ou les intérêts ou encore les valeurs des acteurs impliqués» (1980:83). Quant à la genèse des problèmes sociaux, Rezsohazy estime que "ceux-ci résultent du changement et le suscitent à la fois " (1980:83). Si l'on suit son raisonnement, il y a des problèmes sociaux qui sont permanents en quelque sorte alors que d'autres sont relatifs et occasionnels. Ils sont également différents suivant les époques, les 
lieux, les groupes : " en 1848, on en connaissait d'autres qu'en 1968, la France en affronte d'autres que l'Allemagne, les employés en rencontrent d'autres que les patrons, les gens mariés d'autres que les célibataires et les jeunes d'autres que les vieux» (1980:83). De même, souligne Lenoir, "Ce qui est constitué comme "problèmes sociaux " peut disparaître comme tel alors que les phénomènes qu'ils désignent, subsistent. II en est ainsi, par exemple, de la pauvreté, qui fut, aux États-Unis, un grave problème " social " pendant les années 1930 et disparut dans la décennie 1940-1950, ou encore du racisme qui ne deviendra un problème social que dans les années 1960 " (1989 : 60). Quant à la causalité des problèmes sociaux, elle relève " de multiples facteurs et événements, se situant dans les secteurs les plus différents, qui font fonctionner la société et lui tracent sa trajectoire » (Rezsohazy, $1980: 84$ ).

En 1983, R. Landreville complétait en quelque sorte ces propos en relevant qu'au cours des années, on a constaté que plusieurs événements ou situations perçus comme problèmes pouvaient devenir des situations ou des événements désirables, telle la régulation des naissances; et inversement, certaines situations désirables à l'origine sont maintenant perçues comme des problèmes, tel le travail des enfants (1983:19). D'autres phénomènes sont devenus " neutres " avec le temps (par exemple la " décence » vestimentaire, le divorce, etc.), ou relativement tolérés (comme l'homosexualité et l'avortement). Enfin, il y a les situations où il y a plus ou moins " création" de nouveaux problèmes sociaux (Spector et Kitsuse, 1977). Ainsi, certains auteurs comme Blumer (1971:298) estiment que si l'on veut vraiment comprendre, expliquer les problèmes sociaux, un changement conceptuel est nécessaire. Pour ce dernier, en effet, les approches traditionnelles sont le reflet d'une " grossière incompréhension de la nature des problèmes sociaux " qu'il définit comme "les produits d'un processus collectif de définition » (voir plus loin).

Ceci dit, pour qu'il y ait action par rapport à un problème social, il faut que celui-ci devienne un enjeu. Selon Rezsohazy, un problème devient un enjeu " dès qu'il est reconnu, qu'un acteur le prend en charge, qu'une action se déclenche et que celle-ci mobilise tous les acteurs intéressés " à solutionner le problème $(1980: 85)$. S'en suivent habituellement des luttes de pouvoir entre les divers groupes pour la disposition des "ressources" forcément limitées. Le rôle décisif des acteurs en présence est corroboré par $A$. Beaudoin qui écrivait plus récemment : "On parlera de problème social s'il y a prévision collective que la dégradation d'une situation va se poursuivre selon le processus enclenché et produire des conséquences inacceptables ou de moins en moins acceptables [...] Dans cette perspective, les principes-guides de l'action qui sont définis dans 
toute société pour s'adresser à de telles situations deviennent le point de départ de la politique sociale adoptée. L'action collective formelle qui en résulte prendra différentes formes selon le type de société, les moyens et ressources disponibles ou possibles à développer aux fins de l'action, la conception même de l'action à entreprendre et les groupes en présence» (1987: 16).

Tout en reprenant un peu la perspective de Rezsohazy, De Robertis et Pascal estiment toutefois qu'un problème collectif est souvent insuffisant pour être le point de départ d'une action; il faut aussi « que ce problème soit perçu comme important et vital par les intéressés eux-mêmes [...] II est utile de se rappeler que les problèmes sociaux sont plus ou moins susceptibles de dynamiser les personnes et les groupes selon qu'il s'agit de problèmes ressentis par les intéressés eux-mêmes, des problèmes reconnus par les institutions et/ou autorités, de problèmes découverts par les travailleurs sociaux à partir de leur contact quotidien avec la population. Cette classification a le mérite de nous rappeler qu'une même réalité peut être perçue et analysée de façon différente selon où l'on est situé, et que ces trois types de problèmes doivent être distingués » (1987: 55).

Parmi les différents discours qui marquent la définition d'un problème social, les discours professionnels sont parmi les plus importants. W. Ryan (1972) décrit comme suit la logique de l'intervention sociale : "Premièrement, identifier un problème social. Deuxièmement, étudier ceux qui sont les victimes du problème social et découvrir en quoi ils sont différents des autres en raison des conditions misérables d'existence. Troisièmement, définir cette différence comme la cause du problème lui-même. Enfin, charger un bureaucrate de l'administration d'inventer un programme d'action humanitaire pour corriger les différences" (cité par A. Caillé, 1986 : 28). Les professionnels définissent donc le problème essentiellement comme une déficience, ce qui entraîne presque automatiquement l'individualisation et la division de la personne ainsi que la psychologisation du problème (McKnight, 1977). Dite en termes personnels, l'idéologie professionnelle nous affirme trois choses : vous êtes déficient, c'est vous le problème, vous avez plusieurs problèmes. Replacés dans la perspective des intérêts professionnels, corporatistes et syndicaux, ces postulats deviennent : nous avons besoin de problèmes, nous avons besoin de vous dire lesquels et, finalement, nous sommes la solution à votre (vos) problème(s). Bref, l'idéologie professionnelle se caractérise par une vision du monde " où nos vies et nos sociétés sont traitées comme une série de problèmes techniques [...] C'est une idéologie qui métamorphose le citoyen en client, les communautés en une juxtaposition d'individus déficients" (1977: 18). C'est en ce sens que Spector et Kitsuse (voir 
plus loin) perçoivent "le processus de définition des problèmes sociaux comme étant d'abord relié à des intérêts professionnels engagés dans l'appropriation d'un champ d'intervention " (Manseau, $1988: 42$ ). Et pour $M$. Tachon, la définition d'un " problème social » nécessite habituellement trois conditions : "premièrement, la mise en évidence d'un contexte singulier comme manifestation d'une contradiction générale qui travaille l'ensemble de la société; deuxièmement, un groupe social intégré dans les réseaux de pouvoir, reconnu comme compétent sur le sujet et ayant accès aux instances de décisions locales ou nationales; troisièmement, la légitimité de ce groupe social à inscrire cette question dans le champ des "problèmes " justifiant une intervention " (1985: 178).

Pour Tachon (1985) et Lenoir (1989 : 94), la perspective professionnelle implique aussi une véritable stratégie de gestion des divers problèmes sociaux, par secteurs et spécialités (jeunes, vieux, immigrants, etc.), au sein de laquelle les jeux stratégiques de différents acteurs viennent transformer la perception initiale des problèmes : "Une hiérarchie s'institue : les acteurs dominants investissent dans des problèmes sociaux "solides"; les acteurs mineurs gèrent les problèmes de moindre importance. II y a ainsi une large correspondance entre la place des acteurs dans le champ et la plus ou moins forte légitimité des problèmes sociaux qu'ils gèrent " (Tachon, 1985 : 179). Dans cette perspective, les institutions et les intervenants analysent et décodent le social et identifient les problèmes et les besoins sociaux selon leur logique propre ainsi que selon leurs intérêts respectifs.

Plusieurs auteurs ont insisté sur le fait que chaque problème social a son histoire et se développe selon une série de phases, chaque phase reflétant un changement dans le groupe qui définit le problème, le type de définition qui en est donné et les actions amorcées dans le but de résoudre le problème en question. Les problèmes sociaux sont ainsi perçus non comme des situations statiques mais comme des séquences d'événements.

Ainsi pour Lenoir (1989 : 89) comme pour Bell (1981), l'apparition et le développement d'un problème social s'effectuent en trois étapes principales. II y a d'abord " des transformations qui affectent la vie quotidienne des individus à la suite de bouleversements sociaux divers et dont les effets diffèrent selon les groupes sociaux ». Mais " ces conditions objectives ne donnent naissance à un problème social que lorsqu'il lui est trouvé une formulation publique ». D'où la nécessité d'analyser toute une série de facteurs reliés à cette deuxième étape : travail d'évocation, d'imposition et de légitimation. Finalement, vient la troisième étape, "le processus d'institutionnalisation, qui tend à figer et à fixer les catégories selon lesquelles a été 
posé et résolu le problème au point de les rendre évidentes pour tous " (Lenoir, 1989 : 89). En somme, il faut qu'à des "transformations objectives sans lesquelles le problème ne se poserait pas, s'ajoute un travail spécifique d'énonciation et de formulation publiques, bref, une entreprise de mobilisation "(Lenoir, $1989:$ 77). Et l'analyse de cette mobilisation constitue une dimension importante de l'analyse sociologique des problèmes sociaux.

II semble donc y avoir un relatif consensus quant aux principaux éléments d'un problème social. En effet, la plupart des définitions, mêmes récentes, se recoupent l'une l'autre et la majorité d'entre elles soulignent l'existence de trois conditions essentielles à l'existence d'un problème social, soit la constatation d'une situation-problème, l'élaboration d'un jugement sur celle-ci et le sentiment de pouvoir modifier la situation. La constatation d'une situation-problème est peut-être l'aspect le plus facile à cerner, puisqu'il y a de nombreuses situations (délinquance, pauvreté, alcoolisme, etc.) qui sont jugées " anormales", dont on peut constater les effets et les conséquences. C'est l'étape de la prise de conscience. Toutefois, une situation dans une société n'est jugée comme problème social qu'en vertu des valeurs admises par cette société, ce qui la distingue des autres types de problèmes. Finalement, pour qu'une situation soit considérée comme un problème social, elle doit être perçue comme étant corrigible; sinon il s'agira d'une situation à laquelle on doit s'adapter. C'est l'étape de la réforme ou de la prise en charge du problème par une institution ou une organisation et de la redéfinition de celui-ci en fonction des compétences professionnelles de l'organisation.

Les problèmes sociaux constituent donc autre chose que des situations marginales et isolées de la société où ils existent; bien au contraire, ils sont intimement reliés aux structures de la société qui les définit comme étant des situations-problèmes (Durocher, 1965); ils en sont, en quelque sorte, la contrepartie inséparable. De plus, un problème social est souvent le point de rencontre de perceptions fort différentes et parfois même conflictuelles, émanant de groupes ayant chacun leur analyse des causes du problème et des moyens à prendre pour le corriger. Ils essaieront bien entendu de faire valoir leurs idées de façon à ce que les solutions qui seront adoptées correspondent à leurs intérêts. II nous faut donc approfondir ces différences de perspective analytique. 


\section{Présentation de quelques écoles théoriques d'orientation sociologique dans l'analyse des problèmes sociaux}

C'est aux Américains que, traditionnellement, nous devons la majorité des travaux scientifiques sur lesquels reposent les diverses perspectives qui président à l'analyse des problèmes sociaux. On ne s'étonnera donc pas du recours abondant que nous faisons, dans cette partie du présent article, à la documentation américaine pour l'identification et le développement des principales approches. Ce qui ne nous empêchera pas de signaler, lorsqu'il nous paraîtra opportun de le faire, des contributions françaises et québécoises sur les thèmes à l'étude et ce, particulièrement en raison de leur apport à l'intervention sociale.

Dès le début des années 1960, R. Merton (Merton et Nisbet, 1961), figure dominante de la théorie américaine des problèmes sociaux, en regroupe l'étude sous deux catégories principales : la désorganisation sociale et le comportement déviant. Fonctionnaliste de souche, Merton n'en prône pas moins l'utilisation complémentaire des différentes écoles théoriques existantes pour une analyse plus exhaustive des situations problématiques. C'est ainsi qu'en plus d'approcher l'étude du comportement déviant par la théorie essentiellement fonctionnaliste de l'anomie, il y a ajouté celles de l'association différentielle et de l'étiquetage issues du courant interactionniste, de même que la théorie du conflit social souvent considérée comme un courant de pensée distinct en soi. Nous retrouverons chez plusieurs auteurs cette tendance difficilement réalisable mais fortement recherchée et souhaitée, par les scientifiques américains surtout, d'en arriver à une théorie d'ensemble pour l'analyse des problèmes sociaux.

Horton et Leslie (1971) identifient quant à eux trois perspectives principales : celle de la désorganisation sociale, celle de la déviation culturelle, et celle du conflit de valeurs. De son côté, R. Blum (1970) retient cinq notions pour analyser les problèmes sociaux en service social : le changement culturel (incluant le retard culturel), la désorganisation sociale, le conflit culturel, la déviance ou pathologie sociale, notions auxquelles il ajoute celles des statuts et rôles issus des théories du groupe. Pour sa part, A. Etzioni (1976) propose un regroupement en quatre catégories, à savoir : les approches structuro-fonctionnalistes et de consensus, les approches axées sur le conflit social et l'aliénation, l'interactionnisme symbolique et l'ethnométhodologie, et enfin l'approche néo-conservatrice. G. Ritzer (1986) distingue trois angles de prise pour l'étude des problèmes 
sociaux : le comportement déviant, les problèmes d'inégalités et de conflits sociaux, les problèmes reliés aux progrès humains. Selon qu'il analyse ces situations sous l'angle macrologique des structures, il a recours aux théories du changement social, des tensions sociales et des sous-cultures, tirées du courant structuro-fonctionnaliste. Lorsqu'il considère les situations dans leurs dimensions plus restreintes de déviance individuelle, il se réfère à l'interactionnisme symbolique dont il retient les théories de l'association différentielle et de l'étiquetage, ou encore au courant du behaviorisme en psychologie sociale par le biais de la théorie de l'échange et celle de l'apprentissage social.

Se situant dans une perspective historique, Julian et Kornblum (1986) utilisent, en les reliant à trois courants de la pensée sociologique, une division en six perspectives spécifiques à l'étude des problèmes sociaux : la pathologie sociale et la désorganisation sociale qu'ils relient au fonctionnalisme; la perspective du conflit de valeurs reliée au courant dit du conflit social; la perspective du comportement déviant reliée au fonctionnalisme; l'étiquetage issu de l'interactionnisme symbolique; la perspective institutionnelle qui s'inspire et du fonctionnalisme et du conflit social. Utilisant également une perspective historique, Rubington et Weinberg (1989) présentent six approches spécifiques reliées elles aussi aux différents courants sociologiques mentionnés ci-avant : la pathologie sociale, la désorganisation sociale, le conflit de valeurs, le comportement déviant, l'étiquetage ou perspective interactionniste et la perspective critique.

Du côté français, et adoptant aussi une perspective historique, C. Bachmann et J. Simonin (1981) reprennent les travaux de N. Herpin (1973 : 22) sur les principales problématiques théoriques qui orientent l'action sociale, en Amérique du Nord et en Europe, depuis le début du siècle. Ils démontrent que, depuis son début de professionnalisation, l'action sociale s'est référée à plusieurs modèles théoriques dont les principaux sont: l'École de Chicago, le modèle culturaliste, les approches fonctionnalistes, la problématique interactionniste. Pour les besoins du présent article, et à cause de son lien à l'intervention sociale, nous nous inspirons principalement du cadre de présentation de ces auteurs, cadre auquel nous ajoutons, en guise de complément, la perspective critique et le constructivisme.

\section{L'École de Chicago et le modèle écologique}

Herpin (1973:25), de même que Rubington et Weinberg (1989 : 130), situent durant les années 1920 et le début des années 1930 les bases de développement du modèle de l'École de Chicago, emprunté 
à l'écologie animale (Herpin, 1973 : 25). Selon Bachmann et Simonin (1981: 23), les praticiens et universitaires de cette école de pensée concevaient effectivement l'univers urbain en référence à une métaphore écologique. La ville est divisée en zones naturelles (quartier), les territoires sont délimités, des modes de communications et d'échanges entre communautés s'établissent, des règles de coexistence s'instaurent; bref la ville s'organise selon le principe d'une adaptation mutuelle. Cela correspond à "l'équilibre naturel des espèces » pour le règne animal. Toutefois, certains facteurs, surtout dans les grandes villes, viennent rompre cet équilibre. Surgissent alors des phénomènes de désorganisation : par exemple, la grande mobilité de la population, de forts taux de chômage, des problèmes d'habitat, de criminalité, de délinquance, etc. C'est là qu'entrent en jeu les intervenants sociaux. Comme la ville est source de ruptures et d'exclusions, ils proposent une auto-organisation, sur la base de la communauté. Rubington et Weinberg (1989) soulignent l'insistance que portaient les théoriciens de cette approche à la description plutôt qu'à la théorie. Comme en témoignent leurs études de ce temps sur les hobos, les ghettos, les désordres mentaux et autres types de problèmes, professeurs et étudiants utilisaient la ville de Chicago comme laboratoire naturel pour leurs études. On retrouve des échos de cette perspective, par exemple, dans les propos de S. Alinsky (1976) qui a beaucoup œuvré à Chicago même. Par ailleurs, F. Mourant (1984: 152) souligne que plusieurs auteurs ont appliqué cette perspective à l'étude de la délinquance et ont développé le concept de "zone de délinquance", selon lequel certains milieux produisent une désorganisation sociale qui conduit à la délinquance juvénile.

\section{Le culturalisme}

Herpin (1973 : 28) situe dans la période précédant la seconde guerre mondiale le développement de la pensée culturaliste aux ÉtatsUnis. L'unité théorique des travaux des tenants de cette orientation serait fondée sur "le rapport privilégié qu'ils entretiennent avec la psychanalyse freudienne" (1973:28). Selon le même auteur (1973 : 29), le problème central que posent les culturalistes est celui de la personnalité et le concept clé est celui de la socialisation, c'est-àdire l'intériorisation par les membres d'une société des modèles culturels spécifiques à cette société. Dans cette perspective, ce qui intéresse le chercheur, ce sont les différences culturelles entre les sociétés, les différences dans la " personnalité de base " des individus lorsqu'ils appartiennent à divers milieux culturels, ainsi que les mécanismes de socialisation par lesquels un produit naturel brut, 
quasi identique à l'origine, devient différencié par suite de son appartenance à une culture différente. Cette perspective a été largement utilisée pour expliquer divers problèmes sociaux. Par exemple, plusieurs auteurs ont soutenu que la sous-culture délinquante est une sorte de compromis entre la sous-culture prolétaire de leur milieu d'origine et la sous-culture de la classe, qui est toutefois dominante dans le système scolaire. Ce genre d'explication est vite devenu très populaire : de la pauvreté à l'homosexualité, presque tout a été expliqué en termes de sous-culture.

Associés à cette perspective, il y a ceux qui mettent l'accent sur les conflits de valeurs. Issue de la synthèse des théories américaines et européennes du conflit social (Rubington et Weinberg, $1989: 89$ ), cette approche stipule que les sociétés modernes sont caractérisées par une diversité et une hétérogénéité de valeurs, ce qui aboutit à des divergences qui sont sources de problèmes sociaux. Même si les premiers travaux inspirés de cette approche furent produits dès les années 1920, c'est au début des années 1940, avec les travaux de Fuller et Myers (1941), que s'implante véritablement la formulation de la perspective du conflit de valeurs (Rubington et Weinberg, $1989: 90$ ). Cette formulation, reflétant l'époque (dépression économique suivie de la seconde guerre mondiale), met en évidence non seulement la dimension des valeurs mais aussi celle des intérêts dans les conflits sociaux. C'est dans cette optique que Rubington et Weinberg (1971) abordent les conflits de valeurs du point de vue de luttes émergeant entre groupes sociaux, notamment les luttes sociales et les actions collectives entreprises par les étudiants ou les comités de citoyens depuis les années 1960 et qui ont permis à des individus de se regrouper autour d'attitudes, de valeurs, de droits et d'intérêts partagés. Blum (1970 : 103) estime lui aussi que les conflits de valeurs sont à l'origine des problèmes sociaux parce qu'ils produisent des définitions conflictuelles des conditions sociales considérées comme indésirables et parce qu'ils sont une source de confusion (anomie) qui encourage la déviance personnelle.

Le conflit culturel représente une autre dimension reliée à ce courant de pensée. Une bonne illustration de la théorie générale du conflit culturel est la théorie dite du " retard culturel " (" cultural lag ", W. Ogburn, 1923), élaborée pour expliquer les problèmes sociaux dans les pays en voie de développement en fonction du facteur " innovation technologique ». Blum (1970:60) reprend cette théorie à titre d'hypothèse d'explication des problèmes sociaux. II utilise la notion de changement socioculturel comme source principale des problèmes sociaux. Dans cette perspective, le changement mine les valeurs et provoque de profondes angoisses chez l'individu (1970 : 57). Un autre exemple d'utilisation des conflits de valeurs est 
le modèle proposé par Cohen (1964) pour analyser les problèmes sociaux dans la perspective du service social. Ce modèle est orienté vers l'analyse des valeurs mises en cause et met en lumière le décalage entre les situations de fait et les normes spécialement reconnues (c'est-à-dire la situation souhaitée).

\section{Le fonctionnalisme}

Le fonctionnalisme a été, pendant longtemps, le modèle dominant dans la sociologie américaine. Simplifiée à l'extrême, on peut dire que cette approche conçoit la société comme un être vivant, un organisme dont le tout et les parties sont solidaires. Chaque organe doit remplir sa fonction par rapport au tout organique. Dans la mesure où le fonctionnalisme met l'accent sur l'harmonie et la collaboration, il était presque normal qu'une telle perspective théorique devienne très populaire dans les milieux du service social. Cette perspective conduit à analyser les dysfonctions au niveau des individus ou des groupes. Si le dysfonctionnement est produit par une rupture des solidarités, l'intervention sociale favorisera alors un nouvel apprentissage des rôles afin que l'harmonie ou la collaboration soit rétablie (Bachmann et Simonin, $1981: 25)$. De son côté, Herpin (1973:41) a bien souligné que ce qui caractérise l'analyse fonctionnelle, c'est l'importance attribuée à l'ordre normatif qui constitue l'élément essentiel d'un système social. Au sein d'un système social ainsi défini, le problème de l'acceptation de normes et valeurs institutionnalisées, par des acteurs qui disposent d'une grande part de libre arbitre, et celui de l'imposition de sanctions à ceux qui s'y soustraient, sont primordiaux. II faut donc distinguer entre les acteurs qui acceptent les mêmes lois et règles sociales et ceux qui les refusent et les ignorent.

Pour illustrer cette perspective, on peut recourir à un exemple classique : celui de Merton et Nisbet (1961), intitulé " Contemporary Social Problems ", qui fut maintes fois remanié et réédité par la suite. Ces auteurs distinguent trois grands processus qui ont contribué à la naissance des problèmes sociaux dans les sociétés industrielles : les conflits institutionnels, la mobilité sociale et l'anomie.

Pour ces auteurs, il n'existe pas de dénominateur commun strict pour comparer l'ampleur des problèmes sociaux ni pour indiquer lesquels sont les plus importants. L'ampleur dépend du degré de divergence des conditions sociales par rapport aux standards sociaux qui prévalent dans la société. Quant à l'importance relative des problèmes sociaux, c'est l'importance accordée aux valeurs par la société qui la détermine. Par conséquent, on peut s'attendre à ce que les solutions proposées pour faire face à une situation-problème 
soient divergentes et à ce que le changement représenté par les solutions proposées s'accorde avec les intérêts et les valeurs de certains groupes, au détriment des intérêts et valeurs de certains autres groupes.

L'orientation fonctionnaliste introduit aussi la distinction entre les problèmes sociaux manifestes et les problèmes sociaux latents. Les premiers sont ceux qui sont généralement reconnus comme des problèmes. Les seconds sont des conditions qui ne sont pas largement identifiées comme problèmes, bien qu'en fait elles soient en désaccord avec les intérêts et les valeurs des gens. Ainsi les problèmes sociaux présentent à la fois un aspect subjectif et objectif. Merton et Nisbet (1961) précisent par ailleurs que la perception populaire des problèmes sociaux, même manifestes, est souvent erronée ou inexacte. Il y a deux raisons principales à cela : d'abord nous avons généralement une perception médiatisée des problèmes sociaux et en outre, quelques-unes des formes les plus pénétrantes de désorganisation sociale, comme la pauvreté, ont peu de visibilité publique. Les perceptions populaires des problèmes sociaux ne sont donc pas nécessairement le meilleur guide pour l'évaluation objective de leur dimension et de leur importance. D'autant plus que la perception des problèmes sociaux varie aussi selon la structure des relations sociales. Par exemple, plus la distance sociale entre les victimes d'une catastrophe et les observateurs est grande, moins ces observateurs sympathisent avec les victimes.

Enfin, pour les fonctionnalistes, les problèmes sociaux sont soit des problèmes de désorganisation sociale, soit des problèmes de déviance sociale. La désorganisation sociale est une quelconque condition qui empêche la structure sociale de fonctionner aussi bien qu'elle le devrait pour rencontrer les valeurs et les buts collectifs. Quant au comportement déviant, il implique des écarts significatifs de la norme socialement assignée aux différents statuts et rôles. La structure sociale et culturelle qui permet un comportement conforme et organisé peut aussi créer certaines tendances vers des comportements déviants et de désorganisation sociale. Dans ce sens, les différents problèmes sociaux représentent, pour ainsi dire, les coûts sociaux pour le maintien d'une organisation particulière de la vie sociale. Dans une certaine mesure, il résulte que les problèmes sociaux sont souvent les conséquences non anticipées de l'institutionnalisation de certains comportements sociaux. Donc, les deux facettes, désorganisation et organisation, sont théoriquement inséparables.

Blum (1970: 73) identifie un certain nombre de symptômes généraux de la désorganisation sociale : le formalisme, le déclin du sacré, I'individualisme, etc. Mais pour Merton, l'éclatement du 
système social est plutôt attribuable à un problème de communication entre les membres, l'incompatibilité entre les valeurs et les intérêts de divers groupes, une déficience dans le processus de socialisation et de mauvais aménagements dans les demandes de membres ayant plusieurs statuts occupationnels. Conséquemment, pour remédier au problème social, il est nécessaire de reconstruire un nouvel état d'organisation ou d'équilibre social dans la société.

En ce qui concerne la déviance, Horton et Hermanson (1975) estiment qu'elle peut prendre différentes formes et résulter de différents facteurs. II y a d'abord une inhabileté à suivre les normes généralement aceptées, comme dans la maladie mentale, la dépendance à l'alcool et aux autres drogues, ou une impulsion psychologique interne à commettre l'acte déviant. Dans ces cas, les causes de la déviance sont de nature biologique, psychologique ou physiologique et le comportement qui en découle échappe au contrôle de la personne. Dans d'autres cas, il y a le refus d'accepter les normes généralement reconnues. Dans ces cas, la personne a été socialisée à des normes déviantes.

Chez Merton (1965), l'explication de la déviance prend son point de départ dans le concept d'anomie de Durkheim. En distinguant entre culture et société, il note l'existence, d'une part, d'un système organisé de valeurs qui gouvernent la conduite des individus appartenant au même groupe et, d'autre part, celle de normes et de moyens institutionnalisés, acceptables pour la société, qui règlent l'accès à des buts définis d'après la culture. Or, une tension s'établit entre les buts (culture) et les moyens admis (société). Cette désarticulation de la culture et de la société, où l'une empêche ce que l'autre propose, conduit à une dissolution des normes et à l'établissement de l'anomie : un état social caractérisé par une absence de normes. Par exemple, les couches défavorisées sont induites à partager le "rêve américain " mais ne possèdent pas les moyens légitimes de l'atteindre. Dans ce contexte, la délinquance est une forme d'adaptation "normale » (Mourant, $1984: 153$ ).

Horton (1966) estime que la plupart des théories sociologiques qui abordent l'analyse des problèmes sociaux se rattachent à l'un ou l'autre des grands paradigmes, soit le modèle de l'ordre, soit le modèle du conflit. Les théoriciens de l'ordre privilégient le concept d'anomie alors que ceux du conflit mettent l'accent sur celui d'aliénation. L'une et l'autre théories sont cependant tout autant normatives dans la mesure où elles interprètent les faits à l'intérieur d'une analyse idéologique concernant la nature de l'homme et de la société. Le fonctionnalisme relèverait du modèle de l'ordre dans la mesure où, pour les fonctionnalistes, le problème social est avant tout un problème de socialisation; il s'agit donc, pour le régler, de renforcer l'autorité des impératifs et des interdits collectifs. 


\section{L'approche critique dite " du conflit social "}

Comme le fonctionnalisme, la théorie du conflit social, en sciences sociales, est une approche macrologique qui concentre son attention sur les structures sociales et où la société est vue comme un tout (Ritzer, 1986 : 26). Cependant, au lieu d'étudier les répercussions des différentes structures sociales sur le système entier, les théoriciens du conflit social s'intéressent plus spécifiquement aux façons dont une société est organisée pour servir les intérêts d'une minorité de possédants et puissants aux dépens du reste de la société (Idem). C'est à cette division de la société en deux groupes principaux, polarisés par des intérêts contradictoires, que renvoie le concept " classe sociale». Une dynamique conflictuelle, la lutte des classes, préside aux rapports qu'entretiennent ces deux classes.

L'approche de conflit social, qui anime la perspective « critique » la plus largement utilisée dans l'analyse des problèmes sociaux, prend sa source dans la pensée marxiste qui a inspiré divers courants ayant marqué nombre d'analyses et actions sociales au vingtième siècle. Rubington et Weinberg (1989 : 239) situent surtout durant les années soixante-dix la période principale de l'application de cette perspective à l'étude des problèmes sociaux en Amérique du Nord. Sont souvent cités comme conditions d'émergence de cette approche, des facteurs tels que l'agitation sociale des années soixante aux États-Unis, l'influence des penseurs de l'École de Francfort et de leur critique acérée de la culture du capitalisme avancé, et le développement d'une théorie radicale en criminologie. Comme le signale F. Mourant, " c'est avec la parution des ouvrages collectifs The New Criminology en 1973 et Critical Criminology en 1975, que se manifestent pour la première fois de façon systématique et explicite une critique sociale ayant la criminologie pour base et un questionnement en profondeur des idéologies et des courants philosophiques qui la nourrissent » (1984: 159).

Le modèle du conflit s'oppose à l'ordre de la société parce que ce dernier apparaît comme une stratégie de la classe dominante, une réification des valeurs et des motivations, et une rationalisation du mécanisme de contrôle social. Les tenants des théories du conflit perçoivent la société, non comme un système naturel, mais comme un lieu de conflits politiques continuels entre des groupes ayant des objectifs sociaux différents et une vision du monde opposée (Horton, 1966). Quant aux problèmes sociaux, ils proviennent essentiellement des actions d'exploitation des classes dominantes. Ainsi, ils ne reflètent pas les échecs de l'individu à remplir ses rôles sociaux comme le suppose la tradition de l'ordre, mais au contraire, les incapacités de la société à rencontrer les besoins nouveaux des individus et des collectivités et les conflits de pouvoir à l'intérieur de 
la structure sociale (Etzioni, 1976). Conséquemment, les partisans de cette approche ne portent qu'une faible attention aux caractéristiques innées des individus. Selon eux, la nature humaine est déterminée par les conditions historiques, économiques et sociales dans lesquelles elle se retrouve.

Certains auteurs insistent sur les fonctions positives du conflit : quelquefois il aide à établir l'unité et la cohésion dans les groupes (Coser, 1957); c'est un moyen de résoudre la tension entre les antagonistes et un moyen pour amener le changement dans la société. Les tenants de l'approche du conflit social mettent également l'accent sur le fait que les personnes auront à être "transformées" pour être libérées des dépendances psychologiques et idéologiques à travers lesquelles les anciens régimes ont exercé leur emprise sur les individus. Selon ces théoriciens, les problèmes sociaux existent parce que ceux qui sont au pouvoir ne désirent pas vraiment les résoudre et que des portions significatives de la classe actuelle au pouvoir bénéficient assez directement de l'existence de problèmes sociaux. Seul un " processus révolutionnaire" (et non des "réformes") qui corrigera les conditions d'aliénation, permettra d'assurer la victoire contre les problèmes sociaux.

Pour les théoriciens du conflit, nous dit G. Ritzer (1986:27), le problème social ultime serait le système capitaliste lui-même puisque la plupart des problèmes sociaux les plus spécifiques tirent leur origine du problème majeur de l'inégalité inhérente au système même. Par exemple, les hauts taux de crime seraient attribuables pour une grande part à l'écart immense et infranchissable qui sépare le riche du pauvre, comme l'abus de drogue serait relié aux intérêts des "barons de la drogue " et des politiciens qui tirent avantage de ce commerce. Pour ces théoriciens, les problèmes sociaux portent des enjeux politiques déguisés et ils sont, jusqu'à un certain point, " les enfants naturels" de la structure économique capitaliste et des relations de pouvoir qu'elle génère (Etzioni, $1976: 10$ ).

Pour les tenants de l'approche du conflit social, I'appauvrissement des masses de travailleurs salariés représente le facteur majeur des problèmes sociaux de toute société régie par les règles du capitalisme dont le fonctionnement dépendrait en bonne partie de l'exploitation de la force de travail des masses laborieuses (Etzioni, 1976 : 29). L'aliénation des classes dominées représente aussi une composante majeure des problèmes sociaux. Le rapport compétitif qui résulte du jeu de la concurrence économique crée un monde dominé par la possession matérielle et génère une machine productive qui opprime ceux qu'elle prétend servir. Etzioni expose ici l'idée de Marx selon laquelle, sous le règne du capitalisme, des choses considérées jusque là comme étant inaliénables deviennent des 
objets d'échange et de marchandage, tout objet physique aussi bien que moral étant mis en marché comme biens à être taxés à leur " juste " valeur marchande (K. Marx, cité par Etzioni, 1976 : 11). Et Etzioni de poursuivre que cette déshumanisation serait à la racine même des problèmes sociaux, ces derniers surgissant de la transformation de toute chose précédemment valorisée comme une fin en soi, y inclus le sens même de l'existence humaine, en une instrumentalité, une valeur d'échange, une chose à être négociée, trafiquée contre d'autres choses, d'autres biens sur le marché. C'est pourquoi la principale responsabilité du crime ne revient pas à l'agresseur mais à la structure politique et socio-économique qui le prive d'un standard de vie décent; l'alcoolisme est une évasion à laquelle le travailleur a recours plutôt que de faire face à ses véritables ennemis, l'exploiteur et les tensions sociales générées par un travail routinier et dénué de signification existentielle. De façon générale, même si le lien n'est pas toujours aussi évident entre les conditions de vie et les problèmes sociaux, les tenants de l'approche du conflit social attirent l'attention sur le fait que le comportement déviant découle de problèmes politiques dont la vraie nature est occultée par le traitement que lui réserve la société, traitement inspiré d'un « modèle thérapeutique " plutôt que de mesures de changement social.

\section{L'interactionnisme symbolique et les théories de la réaction sociale et de l'étiquetage}

La perspective interactionniste dans l'étude des problèmes sociaux provient de deux écoles de pensée : l'interactionnisme symbolique et l'ethnométhodologie. Héritiers intellectuels de l'École de Chicago (Etzioni, 1976 : 16), les tenants de ces deux courants de la psychologie sociale américaine centrent leur attention sur le contexte immédiat dans lequel s'inscrit le comportement des individus, objet de leurs études. Une différence subtile distingue les deux groupes. "Le premier, s'inspirant de G.H. Mead (1934), est connu sous le nom $\mathrm{d}^{\prime}$ " interactionnisme symbolique »: interactionnisme parce que la réalité sociale est considérée comme étant formée d'une multitude de petites interactions concrètes entre les personnes; symbolique parce que le langage abstrait de ces interactions leur donne une signification " (Mourant, $1984:$ 156). "Le second courant, l'« ethnométhodologie " est dérivé de la sociologie phénoménologique pour qui la réalité est un objet construit socialement au moyen de définitions émanant d'individus et de groupes, donc non susceptible d'être étudié objectivement " (Idem).

Tirée de ces deux courants de pensée, la théorie de l'étiquetage, désignée aussi sous le nom de perspective interactionniste, re- 
présente un angle d'approche important dans l'étude des problèmes sociaux. Rubington et Weinberg $(1989: 184)$ situent le développement de cette perspective pour le champ des problèmes sociaux à la fin des années cinquante et durant les années soixante, période où, aux États-Unis, l'étude du crime, de la déviance et du contrôle social sont devenues des spécialités en sciences humaines. Des facteurs comme l'extension des concepts, les conflits d'écoles sociologiques et l'insatisfaction devant les théories existantes pour comprendre le comportement déviant (Idem) expliqueraient le recours grandissant à une approche qui constitue en soi une remise en question des autres écoles de pensée.

Comme le précisent Julian et Kornblum (1986:14), les tenants des autres perspectives considèrent, par exemple, que la pauvreté, la délinquance, la toxicomanie, le crime représentent des situations concrètes, définies objectivement comme des problèmes sociaux, dont ils essaient d'identifier les conditions d'émergence. Les théoriciens de la perspective interactionniste, de leur côté, considèrent que les définitions socialement acceptées de la déviance ou des problèmes sociaux sont subjectives; aussi, leur tâche consistera-t-elle à tenter d'expliquer pourquoi et sous quelles conditions certains actes et certaines situations en viennent à être définis ou jugés problématiques ou déviants. Cette approche suscite donc "un renversement de la question, qui ne se pose plus désormais au sujet de la personne du déviant, mais plutôt au sujet de la réaction sociale " (Mourant, 1984 : 156).

Plusieurs auteurs illustrent bien cette position. Jones, Gallagher et McFalls (1988 : 23) avancent qu'en tant que processus social, le comportement déviant se présente logiquement en deux stades : d'abord, l'acte de violation de la norme sociale et ensuite, la réaction sociale à cet acte qui décide ce qu'est la déviance et à qui accoler l'étiquette de déviant. Ainsi, un comportement inapproprié peut être vu comme une excentricité si le déviant est riche, et comme un désordre mental s'il ne l'est pas (Scheff, 1975, cité par Jones et al., 1988 : 23). Dès lors, la déviance n'est pas une qualité inhérente, intrinsèque au comportement, mais le résultat de l'interaction entre la personne qui pose l'acte et les autres qui y réagissent. On voit donc que le courant interactionniste propose un changement d'objet : " ce n'est plus l'étude des causes de la déviance qui importe, mais bien le processus par lequel certaines formes de comportement, et les personnes qui y sont associées, en viennent à être perçues, définies et traitées par d'autres comme déviantes en général ou délinquantes en particulier» (Mourant, $1984: 157)$.

Bachmann et Simonin (1981 : 25) insistent eux aussi sur le fait que les interactionnistes s'interrogent sur les situations qui font pro- 
blème, en ajoutant que la désignation d'un individu comme déviant mène elle-même à une accentuation de la déviance. L'idée s'en dégageant est que la société, tout en voulant contrôler la déviance, ultimement, la produit. Fondamentalement d'ailleurs, comme l'a souligné H. Manseau (1988: 9), l'identification des problèmes sociaux, dans ce modèle, découle donc davantage des conceptions propres à des groupes ou personnes qui sont parvenus à s'approprier une question sociale comme champ d'action ou de compétence. Elle rejoint par là Rubington et Weinberg (1989: 189) qui, s'intéressant aux conditions qui favorisent la réussite de l'étiquetage, font remarquer que l'étiqueteur est habituellement dans une position où il tire avantage du fait de décerner une étiquette. Très souvent, poursuivent les mêmes auteurs, l'étiquetage est fait par quelqu'un dont $c^{\prime}$ est la tâche d'appliquer des caractéristiques définitionnelles (par exemple les agents de contrôle social, les journalistes) et le faire est souvent un signe de succès dans sa fonction.

Ainsi, pour les interactionnistes, le sens donné à toute situation est un construit, la désignation officielle des problèmes sociaux ou des crimes ne relevant pas tant d'un consensus que de l'interprétation de ceux qui interviennent dans la définition des lois et leur mise en application. Dans la même logique, les statistiques enregistrées au sein des appareils de contrôle ne témoignent pas tant de l'ampleur objective d'une question que de l'action définitionnelle et de ses aboutissements. Les interactionnistes estiment en effet que l'incidence statistique d'un problème peut surtout se comprendre à partir des activités définitionnelles des personnes qui ont concouru à la reconnaissance de ce problème (Kitsuse et Cicourel, 1963). De même, les tenants de la perspective de la réaction sociale contestent I'orientation étiologique (Mourant, 1984 : 145). Selon eux, présenter la déviance comme une donnée ontologique, antérieure à la réaction sociale, constitue une inversion logique puisque le déviant ou le délinquant est un " construit social ». De plus, analyser les causes de cette déviance indépendamment de l'étude de la dynamique sociale ayant contribué à l'émergence des normes morales, sociales ou pénales, c'est prendre les reflets des choses pour la réalité (Chamboredon, 1971).

Par exemple, lors d'un premier vol, un jeune peut rapidement être "étiqueté " délinquant par la police (Bachmann et Simonin, 1981 : 25). Ainsi, il peut, par la suite, en venir par lui-même à adopter la conduite qu'on attend de lui. Conséquemment, la déviance est le produit conjoint des institutions qui définissent qui est déviant, et des acteurs sociaux qui s'enferment dans ces définitions; l'acte de déviance n'est plus le moment où l'individu transgresse la loi mais le processus complexe au cours duquel il en vient à être désigné comme 
déviant (Becker, 1966). Il importe donc de distinguer les phases de ce processus et d'analyser les conditions sociales qui favorisent ou non la déviance. Parmi les conditions sociales, il y a, rappelons-le, ce que les interactionnistes nomment la réaction sociétale. L'infraction à la norme $n^{\prime}$ inaugure une carrière de déviant qu'à la condition que cette infraction soit socialement perçue comme telle. Ce n'est pas la première infraction qui est déterminante, mais le déclenchement des mécanismes sociaux de désignation. Pour ce courant de pensée donc, " ce qu'il importe de découvrir par l'analyse des problèmes sociaux, ce sont les forces sociales qui sont productrices de sens. L'interactionnisme symbolique suppose que l'objectivité n'existe pas. Même si la misère humaine a des fondements réels, l'importance accordée à tel ou tel problème particulier, de même que les représentations qui l'entourent, dépend des individus qui ont réussi à imposer leur définition du problème dans un contexte social donné " (Manseau, $1988: 42)$.

En somme, dans la perspective interactionniste, si l'on veut comprendre la déviance, il ne faut partir ni des individus, ni des comportements des individus, ni même des règles qui sont éventuellement transgressées, mais des situations dans lesquelles il peut advenir que soient désignés des déviants. II n'y a, en effet, rien de prédéterminé, et la distribution éventuelle des désignations de déviants est le produit émergeant de l'interaction. Ceci rompt avec la façon traditionnelle d'envisager un problème social, dans la mesure où le regard se porte désormais sur les définisseurs du problème, sur la définition qu'ils proposent et sur les solutions proposées. Les définisseurs deviennent ainsi une partie même du problème. C'est pourquoi le processus d'étiquetage ("labelling ») est au centre de la perspective interactionniste.

\section{Le constructivisme}

Plus en continuité qu'en rupture avec le courant précédent, les constructivistes mettent l'accent sur les processus de construction sociale des problèmes sociaux. Pour ces derniers, un problème social est le résultat des démarches d'individus ou de groupes concernant des demandes de modification de certaines conditions sociales (Spector et Kitsuse, $1977: 75$ ). Cette perspective déplace l'accent traditionnellement mis sur les conditions objectives vers le processus par lequel se construisent les définitions de problèmes sociaux.

L'existence d'un problème social dépend de l'existence des groupes qui définissent une condition comme problématique, veulent y remédier et agissent dessus. Il y a deux types de groupes : ceux directement touchés par la situation problématique et ayant intérêt à 
la transformer et ceux qui ne sont pas impliqués mais dont la situation heurte les valeurs. Les groupes sociaux peuvent donc être guidés dans leur action, soit par leurs intérêts, soit par leurs valeurs. Les auteurs élaborent ainsi un nouveau cadre d'analyse des problèmes sociaux comportant trois éléments de base; une théorie des intérêts : les groupes comme les individus cherchent à défendre leurs intérêts économiques, sociaux, etc.; une théorie de l'indignation morale : les groupes dénoncent certaines conditions comme problématiques, car elles heurtent leur sens des valeurs, indépendamment de leur intérêts; une théorie de l'histoire naturelle : les problèmes sociaux n'émergent pas d'une situation statique ou d'un événement spontané, mais de séries d'activités évoluant et s'influençant les unes les autres (Schneider, 1985).

Cette approche remet nécessairement en question la notion de consensus social et conduit Spector et Kitsuse (1977) à proposer une autre définition du problème social. Pour eux, un problème social est constitué par " les démarches d'individus ou de groupes formulant des griefs et des revendications à propos de certaines conditions supposées exister " (1977: 75). Une démarche de revendication est une forme d'interaction entre deux ou plusieurs protagonistes sociaux à l'intérieur de laquelle une partie demande à l'autre que soit fait quelque chose par rapport à une certaine condition sociale jugée insatisfaisante afin de l'améliorer ou de la changer. Conçues comme telles, les démarches de revendication font partie intégrante de la vie politique et sociale et tous ceux qui s'y impliquent participent de facto au processus de définition des problèmes sociaux. Conséquemment, les auteurs étudient les problèmes sociaux en partant des individus qui parviennent à les faire émerger en tant que problèmes, et en mettant l'accent sur les intérêts des individus ou des groupes qui participent à la définition de ces problèmes.

Ils proposent également un modèle d'analyse de l'évolution des problèmes sociaux en quatre étapes principales. La première comprend " les tentatives collectives pour remédier à une condition perçue et jugée choquante et indésirable par certains groupes. Les tentatives pour transformer des problèmes privés en litiges publics et les éventualités de ce processus de transformation, constituent les activités initiales des problèmes sociaux " (Spector et Kitsuse, 1973 : 148). La deuxième étape commence avec l'approbation de ces revendications par " des agences gouvernementales ou autres institutions officielles influentes ». En effet pour passer à la phase suivante, un problème social doit concerner et être pris en charge par " une institution [...] afin de gérer les revendications et les plaintes relatives à la condition en question »(Spector et Kitsuse, 1973 : 154). La phase 3 commence quand les participants considèrent la réponse 
officielle comme problématique. La phase 4 prend naissance quand les revendicateurs " affirment qu'il n'est plus possible "de fonctionner dans le système" $[\ldots]$ et quand ils tentent d'élaborer des institutions alternatives " (Spector et Kitsuse, $1973: 156)$. Selon ces derniers, il n'existe pas de relation causale simple entre une condition sociale insatisfaisante et la mise en marche des réponses institutionnelles. Ils insistent sur le fait que les problèmes sociaux résultent du travail de plusieurs acteurs sociaux (tels que journalistes, médecins, politiciens, travailleurs sociaux et organisateurs syndicaux) et que plusieurs aspects des problèmes sociaux peuvent être abordés à travers l'étude des personnes qui participent aux diverses étapes du processus de construction de la réalité des problèmes sociaux.

Depuis le début des années 1980, plusieurs auteurs ont repris ce thème de la " construction » des problèmes sociaux. Ainsi Bachmann et Simonin (1981:21) soulignent que I'on doit admettre que le concept de problème social est relatif et qu'il est construit. De même, Martin et Chopart affirment sans ambages : "Les problèmes sociaux n'existent pas comme tels. Ils font l'objet de processus de construction " (1988: 85). Même analyse chez Hulsman (1981) et Landreville (1983 : 18) qui soulignent que dans le champ criminologique, on envisage souvent une situation-problème en termes d'infraction ou de crime et ce faisant, on commet, selon eux, une erreur fondamentale qui "consiste en ce que comportement et infraction sont considérés comme le point de départ. On prend ces faits comme des données et non comme des construits» (1981:12).

Selon Martin et Chopart (1988: 85), le travail scientifique dans l'analyse des problèmes sociaux doit consister "à comprendre comment une société donnée envisage, à un moment donné, la nécessité de régulations, d'interventions politiques, collectives, réglementaires, afin de résoudre des zones de tensions devenues incontournables ». II faut aussi procéder à un important travail de "déconstruction" portant notamment "sur la mise en œuvre des systèmes $d^{\prime}$ intervention, sur les cloisonnements qu'ils induisent, leur capacité de réifier tel ou tel aspect de la vie sociale : la pauvreté est isolée du chômage, isolée de la vie familiale, etc. » (Idem). Bref, il faut faire apparaître les interconnexions complexes qui relient "ces problèmes sociaux, leur catégorisation et leur mode de traitement » (1988: 86).

Tachon s'attarde de son côté sur l'idée qu'étant essentiellement le résultat (ou le produit) "de constructions historiques", les problèmes sociaux "apparaissent comme des notions relatives, faisant l'objet de réinterprétations par les agents et les institutions dans leurs stratégies pour se partager les moyens symboliques, économiques et techniques de l'action sociale » $(1985: 177)$. Ce 
processus de réinterprétation constitue une véritable " mise en scène " du problème social. Par exemple, les problèmes de la délinquance juvénile ayant été identifiés comme des problèmes de sécurité sous la pression convergente de groupes divers (policiers, magistrats, enseignants, politiciens, etc.), on a pu voir l'État désigner l'insécurité liée à la délinquance juvénile comme " problème social " et dans le même mouvement, définir une méthodologie d'intervention. Ainsi, l'intervention sociale se construit presque toujours à partir d'un même scénario : "un problème social " légitimé par des références politiques et techniques génère des institutions qui mobilisent des investissements et des personnels spécialisés. Les institutions et les personnels spécialisés jouent alors avec la manifestation publique du "problème "; ils proclament l'urgence de la question, justifiant ainsi leur présence. Cette situation est amplifiée par la concurrence et la surenchère entre les différentes instances du " travail social ", les diverses prestations assurées par les institutions se présentant comme des réponses originales à un problème social identifié (1985: 179).

Guillemard (1986) et Lenoir (1979) ont bien montré, dans leurs travaux sur le " troisième âge ", les mécanismes socio-historiques de l'émergence des problèmes sociaux. Ce dernier, notamment, montre que "l'invention du troisième âge " correspond d'abord à un problème de solidarité intergénérationnelle qui prend son origine dans l'organisation industrielle et dont l'effet est d'introduire une gestion différenciée de cette population; cette situation génère à son tour l'apparition d'agents spécialisés dans la gestion de la population en question et l'émergence de mouvements de revendications chez cette dernière. De fait, la répartition de l'existence humaine en " âges de vie " avec son cortège de problèmes sociaux, est tout à fait arbitraire. Beaucoup de travaux historiques ont pointé le relativisme des " classes d'âge ", tels les travaux de Ariès (1973), Chamboredon et Prévot (1973) sur l'apparition des notions d'enfance et d'adolescence.

Au Québec, un certain nombre d'études se sont inspirées de la perspective constructiviste. Par exemple, K. Fahmi (1987) s'est attaché à reconstituer les événements qui ont concouru à l'émergence de la prostitution juvénile en tant que problème social au Québec. Certes, ce phénomène n'est pas nouveau chez nous tout comme ailleurs. Toutefois, ce n'est que depuis la fin des années 1970 qu'on s'interroge sur cette réalité en tant que problème social. Plusieurs intervenants sociaux ont participé aux débats sur cette question, qui bénéficièrent $\mathrm{d}^{\prime}$ une large diffusion médiatique au cours des dernières années. À partir de cet exemple, l'auteur affirme que les médias jouent un rôle très important dans la définition et la construction des problèmes sociaux. La construction du problème des jeunes dits prostitués s'est 
donc élaborée autour de différentes scènes. Le même thème fut alimenté par l'arrivée de nouveaux acteurs sociaux (les policiers, les commissions d'enquête, etc.). En bout de ligne, la définition du problème, conclut Fahmi, s'appuie sur un jugement moral (protection et punition) qui tient peu compte de la réalité socio-économique des jeunes vivant cette condition.

Sensiblement dans la même perspective, H. Manseau (1988) a analysé les processus de définition et de prise en charge institutionnelle du problème de l'abus sexuel au Québec. Selon elle, la présence d'un fort mouvement de protection de la jeunesse qui a engendré la mise sur pied de tout un dispositif d'intervention a eu un impact direct sur la définition de l'abus sexuel et sur les représentations que suscite ce phénomène $(1988: 41)$. Tout cela amène l'auteure à conclure que l'abus sexuel " est une forme de construit institutionnel et professionnel comme c'est le cas pour tous les problèmes sociaux qui s'élaborent à partir d'un discours extérieur à celui des personnes faisant l'objet d'un contrôle étatique " (1988 : 46). Elle s'interroge également sur le fait que « les conceptions les plus répandues sur l'abus sexuel ont été élaborées dans un contexte éloigné des situations particulières vécues par les jeunes victimes. Des intérêts liés à la prise en charge du problème semblent contribuer à faire en sorte que cette question soit traitée sous un angle qui ne correspond peut-être pas à l'intérêt des jeunes victimes " (1988: 41).

\section{Conclusion}

II faut rappeler notre objectif de départ. II s'agissait d'abord de tenter de clarifier la notion de problème social et ensuite, d'introduire brièvement un ensemble de perspectives d'analyse de ce concept. Au terme de cet exercice, il nous faut constater qu'un débat a marqué ces divers courants, c'est celui des rapports entre les dimensions objectives et subjectives des problèmes sociaux. De sorte que l'on est maintenant en droit de se demander : mais est-ce dire que tout est subjectif, qu'il n'y a pas de situations, d'événements, de comportements réellement négatifs ou problématiques ? Et avec d'autres, nous répondons : évidemment non. Ainsi, P. Landreville précise : "Même si nous maintenons que dans une démarche sociologique nous devons nous attarder aux situations perçues et définies comme problèmes, déviances, etc., nous croyons que, du point de vue éthique, il y a des situations, des conditions négatives [...] que nous devons tenter de contrôler, d'atténuer, d'éliminer par des politiques sociales adéquates. En premier, il y a des situations d'exploitation, de 
domination et des besoins fondamentaux (vie, sécurité, ...) qui ne sont pas respectés. II y a aussi d'autres besoins (plus subjectifs ?) que l'on juge nécessaires, indispensables dans une société historique donnée (exemple : les droits de la personne, l'égalité, la justice) que I'on voudra utiliser comme critères des situations-problèmes. Mais, quelle que soit la "réalité » ou "l'objectivité » des situationsproblèmes, il faut bien réaliser qu'en termes de politique sociale, il faudra que des groupes jugent ces situations-problèmes et qu'ils aient le pouvoir d'imposer leurs définitions et leurs solutions. II faut rappeler que, même si nous affirmons qu'il y a des situations qui posent réellement des problèmes, cela ne signifie pas qu'il y ait ou qu'il puisse y avoir de "vrais" crimes. Le crime sera toujours un construit juridico-politique, puisqu'il ne s'agit que d'une catégorie de situations dites problèmes, celles que l'on croit devoir gérer ou contrôler par des peines imposées par l'État» (1983: 26). Et F. Mourant de conclure : "Que le crime soit une construction politicojuridique on ne peut qu'en convenir, mais il demeure que certaines formes de comportements sont nuisibles, dangereux et causent de sérieux torts aux autres» (1984: 166).

En somme, nous avons montré qu'en service social comme en sociologie, il existe divers codes de perception, de définition et de traitement des problèmes sociaux. Selon J. Horton (1966), pendant longtemps l'étude des problèmes sociaux a été abordée à partir de deux modèles principaux : celui de l'ordre et celui du conflit. Chaque perspective interprétait les faits à partir d'un contexte idéologique particulier. Ces discours masquaient toutefois leur propre normativité. $D^{\prime}$ une façon générale, on a souvent recours à une terminologie de l'évidence, et la définition d'une situation sociale comme étant un problème social est assujettie aux normes véhiculées par un ou plusieurs auteurs. Même si maintenant les modèles interprétatifs sont plus nombreux, il n'en demeure pas moins que le débat sur I'interprétation subjective d'une réalité dite objective demeure. Dans un tel contexte, il s'avère important d'expliciter clairement sa propre perspective d'analyse et de la situer par rapport aux autres.

\section{Références}

AlinSkY, S. (1976). Le manuel de l'animateur social. Paris : Seuil.

ARIÈs, P. (1973). L'enfant et la vie familiale sous l'ancien régime. Paris : Seuil.

BACHMANN, C. et J. SIMONIN (1981). Changer au quotidien : une introduction au travail social. (2 tomes) Paris : Éd. Études vivantes.

BARBIER, R. (1978). "La tête au ciel et les pieds sur la terre", Informations sociales, Paris, $\mathrm{n}^{\text {os }} 3-4: 31-40$. 
BeAudoin, A. (1987). Le champ des services sociaux dans la politique sociale au Québec. Synthèse critique $n^{\circ} 38$, Commission d'enquête sur les services de santé et les services sociaux. Québec : Les Publications du Québec.

BECKER, H.S. (1986). Outsiders. Paris : Éd. Métaillé. (Traduction de Studies in the sociology of Deviance, 1963).

BECKER, H.S. (1964). The Other Side : Perspectives on Deviance. New York : The Free Press.

BeCKer, H. (1966). Social Problems. A Modern Approach. New York, London, Sydney : John Willey \& Sons Inc.

BeLL, R. (1981). "An Anatomy of Social problems » : 3-29, dans Contemporary Social Problems. Illinois : The Dorsey Press. (Résumé et traduction par Marcelle Laforest, Université Laval, Québec, 1988, 5 p.).

BLum, R. (1970). Dimensions sociologiques du travail social. Paris : Éd. Le Centurion.

Blumer, H. (1971). "Social Problems as Collective Behavior », Social Problems, vol. $18: 298-306$.

CAIllé, A. (1986). Splendeurs et misères des sciences sociales, Esquisses d'une mythologie. Genève : Droz.

CAStel, R. (1981). La gestion des risques. Paris : Éd. de Minuit.

Chamboredon, J.C. (1971). "La délinquance juvénile : essai de construction d'objet ", Revue française de sociologie, vol. 12 : 335-377.

Chamboredon, J.C. et J. Prévot (1973). "Le métier d'enfant ", Revue française de sociologie, vol. 14, $\mathrm{n}^{\circ} 3$.

COHEN, N.E. (1964). Social Work and Social Problems. National Association of Social Workers, New York.

Commission d'enquête sur les services de santé et les services sociaux (1988). Rapport. Québec : Les Publications du Québec.

COSER, L.A. (1957). "Social Conflict and Social Change", British Journal of Sociology, vol. 8 : 197-207.

DeBuYST, C. (1973). "Criminologie de l'étiquetage et criminologie du passage à l'acte ", Annales internationales de criminologie, vol. 12, $\mathrm{n}^{\text {os }} 1-2: 282-290$.

De Robertis, C. et H. PASCAL (1987). L'intervention collective en travail social. Paris : Le Centurion.

Durocher, P. (1965). Les problèmes sociaux. Texte miméo (10 p.). École de service social, Université de Montréal.

EtZIONI, A. (1976). Social Problems. New Jersey : Prentice-Hall Inc. (Résumé et traduction par Marcelle Laforest, Université Laval, Québec, 7 p.).

FAHMI, K. (1987). La prostitution des mineurs : construction d'un problème social. Bureau Consultation-Jeunesse (BCJ), Montréal.

Fuller, R.C. et E. MYers (1941). "Some Aspects of a Theory of Social Problems ", American Sociological Review, vol. 6, nº 1 : 24-32.

Goffman, E. (1975). Stigmate, les usages sociaux des handicaps. Paris : Minuit.

Guillemard, A.M. (1986). Le déclin du social. Paris : PUF.

HeRPIN, N. (1973). Les sociologues américains et le siècle. Paris : PUF.

HORTON, P.H. et G.R. LesLIE (1965). The Sociology of Social Problems ( $3^{\mathrm{e}}$ édition). New York : Appleton-Century-Crafts.

HORTON, J. (1966). "Order and Conflict Theories of Social Problems as Competing Ideologies", American Journal of Sociology, vol. 71, n 6 : 701-713. 
HORTON, P.H. et G.R. LeSLIE (1971). Studies in the Sociology of Social Problems. New York : Appleton-Century-Crafts.

Horton, R. et R. HeRmanson (1975). Social Problems : 13-21. Irwin Inc., Illinois. (Résumé et traduction par Marcelle Laforest, Université Laval, Québec, 1986, 7 p.).

HULSMAN, L. (1981). "Une perspective abolitionniste du système de justice pénale et du schéma d'approche des situations problématiques ": 7-16, dans C. Debuyst (éd.), Dangerosité et justice pénale, Coll. Déviance et Société, Genève.

Jones, B., B. Gallagher et J. McFalls (1988). Social Problems, issues, opinions and solutions. New York: McGraw-Hill Inc.

Julian, J. et W. Kornblum (1986). Social Problems : 2-26. Prentice-Hall, Englewoods Cliffs, N.J. (Résumé et traduction par Marcelle Laforest, Université Laval, Québec, 1988, 2 p.).

Julian, J. et W. Kornblum (1986). Social Problems. (5e éd.). Prentice-Hall, Englewood Cliffs, N.J.

Kallen, D., D. Miller et A. Daniels (1968). "Sociology, Social Work and Social Problems ", American Sociologist, vol. 3, n 3 : 235-240.

Kitsuse, J. et A. Cicourel (1963). "A note of the uses of official statistics ", Social Problems, vol. $12:$ 131-139.

KITSUSE, J. et M. SPECTOR (1973). « Toward a sociology of social problems : Social conditions, value-judgments and social problems ", Social Problems, vol. $20: 407-419$.

KITSUSE, J. et M. SPECTOR (1974). "Social problems and deviance : some parallel issues ", Social Problems, vol. 22, $n^{\circ} 5$ : 584-594.

KITSUSE, J.E. (1980). "Coming out all over : Deviants and the Politics of social problems ", Social Problems, $\mathrm{n}^{\circ} 1: 1-13$.

LALONDE, M.L. (1976). Modèle de pratique du service social personnel en milieu scolaire. Rapport de recherche, École de service social, Université de Montréal.

Landreville, P. (1983). Normes sociales et normes pénales. Les Cahiers de l'École de Criminologie, Université de Montréal, Montréal.

LASKIN, R. (1965). Social Problems : A Canadian Profile. Toronto : McGraw-Hill.

LaUer, R.H. (1976). "Defining Social Problems: Public and Professional Perspective ", Social Problems, vol. 24, n $1: 122-130$.

Lemert, E.M. (1967). "Social Structure, Social Control and Deviation » : 3-31, dans E.M. Lemert, Human Deviance, Social Problems and Social Control. Prentice-Hall.

LENOIR, R. (1979). "L'invention du troisième âge (ou la constitution du champ des agents de gestion de la vieillesse) ", Actes de la recherche : 26-58.

LENOIR, R. (1989). "Objet sociologique et problème social »: 53-100, dans P. Champagne et al., Initiation à la pratique sociologique. Paris : Dunod.

Lopata, H. (1984). "Social Construction of Social Problems over time », Social Problems, vol. $31, \mathrm{n}^{\circ} 3: 249-272$.

MALherbe, N. (1977). "Les tendances récentes de la sociologie de la déviance aux États-Unis : origine et orientations générales", Revue Suisse de sociologie, vol. 2 : 145-184.

MaNIS, J.G. (1974a). "The Concept of social problems : Vox Populi and sociological analysis », Social Problems, vol. 21 : 305-315. 
MANIS, J.G. (1974b). "Assessing the seriousness of social problems ", Social Problems, vol. 22 : 1-15.

MANSEAU, H. (1988). "La définition ou la fabrication de l'abus sexuel d'enfants au Québec ", Revue internationale d'action communautaire, 19/59 : 41-47.

MARTIN, C. et J.N. ChOPART (1988). "Derrière l'éclatement : la permanence de la question sociale", Revue internationale d'action communautaire, 20/60 : 79-89.

MCKNIGHT, J. (1977). "Le professionnalisme dans les services : un secours abrutissant ", Sociologie et Sociétés, vol. 9 : 7-18.

MeAd, G.H. (1934). Mind, Self and Society. Chicago : University of Chicago Press.

MerilL, F.É. (1948). "The Study of Social Problems", American Sociological Review, vol. 13, n $3: 251-259$.

Merton, R.K. (1961). "Social Problems and Sociological Theory " : 697-737, dans R.K. Merton et R. Nisbet, Contemporary Social Problems. New York : Harcourt Inc.

MERTON, R.K. (1965). "La prédiction créatrice ", dans Éléments de théorie et de méthode sociologique. Paris : Plon.

Merton, R.K. et R. Nisbet (1961). Contemporary Social Problems ( $2^{e}$ éd.). N.Y. : Harcourt, Brace and World.

Merton, R.K. et R. NisBet (1976). Contemporary Social Problems. N.Y. : Harcourt Brace Inc. (Résumé et traduction par Marcelle Laforest, Université Laval, Québec, 1986, 5 p.).

Milss, C.W. (1963). "The Ideology of Social Pathologist»: 525-552, dans Power, Politics and People. New York : Ballantyne Books.

Milus, C.W. (1968). L'imagination sociologique. Paris : F. Maspero.

MOURANT, F. (1984). "Déviance et délinquance : une revue des notions", Service social, vol. 33, $\mathrm{n}^{\text {os }} 2-3: 145-170$.

NeubeCK, K.J. (1980). Social Problems, A critical Approach. Glenview, Illinois : Scott, Foresman Co.

Normandeau, A. (1966). "Action sociale et recherche au Québec ", Cité Libre, Montréal, vol. 16, $\mathrm{n}^{\circ} 85: 18-23$.

OGBurN, W. (1923). Social change. New York.

Pelletier, M. (1978). Les fonctions de la sécurité sociale : l'idéologie. Montréal.

Perron, J. (1986). Administration sociale et services sociaux. Chicoutimi : G. Morin Éditeur.

Pineault, R. et C. Daveluy (1986). La planification de la santé : concepts, méthodes, stratégies. Montréal : Les éditions Agence d'Arc Inc.

RezsohazY, R. (1980). "Itinéraires pour l'étude du changement social ", Revue de l'Institut de Sociologie, vol. $1: 80-94$.

RezsohaZY, R. (1982). "Aspects théoriques et méthodologiques de l'étude du changement culturel »: 51-61, dans Collectif (sous la direction de G. Pronovost), Cultures populaires et sociétés contemporaines. Québec : PVQ.

Ritzer, G. (1986). Social Problems ( $2^{\mathrm{e}}$ éd.). New York : Random House.

Rubington, E. et M.S. WeinberG (1971). The Study of Social Problems : five Perspectives. New York : Oxford University Press.

Rubincton, E. et M.S. Weinberg (1973). The Study of Social Problems : five Perspectives. New York : Oxford University Press. 
Rubington, E. et M.S. Weinberg (1989). The Study of Social Problems : six Perspectives ( $4^{\mathrm{e}}$ édition). New York/Oxford : Oxford University Press.

SCHNeIder, J.W. (1985). "Social Problems Theory : The Constructionist View", Annual Reviews Sociology, vol. 11 : 209-229.

SPECTOR, M. et J. KITSUSE (1973). "Social Problems : A re-formulation », Social Problems, vol. 21, $\mathrm{n}^{\circ} 2: 145-159$.

Spector, M. et J. Kitsuse (1977). Constructing Social Problems. Menlo Park, Calif. : Cummings Publishing Co.

SPeCtor, M. et G. CASAdamont (1978). "Profils épistémologiques en criminologie comparée ", Déviance et société, vol. 2, n $4: 349-364$.

TACHON, M. (1985). "Travail social et gestion des problèmes sociaux » : 177 187, dans Lectures sociologiques du travail social (sous la direction de F. Bailleau, N. Lefrancheur et V. Feyre). Paris : Éd. Ouvrières.

VAN OUtRIVE, L. (1973). "Stigmatisation : un prolongement de l'analyse criminologique? ", Revue de droit pénal et de criminologie, vol. 53, $n^{\circ} 4$ : 363-384. 